University of Tennessee Health Science Center

UTHSC Digital Commons

\title{
Effects of Tricalcium Phosphate Coated Titanium on Adjacent Early Bone Formation
}

Bristol Williams

University of Tennessee Health Science Center

Follow this and additional works at: https://dc.uthsc.edu/dissertations

Part of the Periodontics and Periodontology Commons

\section{Recommended Citation}

Williams, Bristol , "Effects of Tricalcium Phosphate Coated Titanium on Adjacent Early Bone Formation" (2007). Theses and Dissertations (ETD). Paper 291. http://dx.doi.org/10.21007/etd.cghs.2007.0350.

This Thesis is brought to you for free and open access by the College of Graduate Health Sciences at UTHSC Digital Commons. It has been accepted for inclusion in Theses and Dissertations (ETD) by an authorized administrator of UTHSC Digital Commons. For more information, please contact jwelch30@uthsc.edu. 


\title{
Effects of Tricalcium Phosphate Coated Titanium on Adjacent Early Bone Formation
}

\begin{abstract}
Dental implants are the gold standard of restorative dentistry today. Implants have been used for several years in Orthopedics. Dental implants are an important treatment option for several patients today. When a patient is fully or partially dentate, they are given the opportunity to restore their dentition in ways similar to their natural oral cavity. The past literature states that titanium is one of the first metals used in implant placement. There have been many improvements made on this material. Most modifications have been used in order to promote more bone growth around these implants. Several different coating materials have been used to increase bone formation. Titanium ( $\mathrm{Ti}$ ) surfaces have been coated with hydroxyapatite (HA), plasma spray, and several blasting techniques have been implemented. Tricalcium phosphate (TCP) is a coating that has been used for several years. Due to its porosity and likeness to natural bone, many feel that it is an ideal coating for both dental and orthopedic titanium implants. We believe that TCP coated titanium increases the amount of osteoblastic cell attachment and early bone producing biomarker proteins in osteoblastic cells when compared to HA coated titanium and titanium only materials.
\end{abstract}

\section{Document Type}

Thesis

\section{Degree Name}

Master of Dental Science (MDS)

\section{Program}

Periodontology

Research Advisor

Joo L. Ong, Ph.D.

\section{Keywords}

Tricalium phosphate, osteoblasts, titanium, alkaline phosphatase, osteocalcin

\section{Subject Categories}

Dentistry | Medicine and Health Sciences | Periodontics and Periodontology 
Effects of Tricalcium Phosphate Coated Titanium on Adjacent

Early Bone Formation

\author{
A Thesis \\ Presented for \\ The Graduate Studies Council \\ The University of Tennessee \\ Health Science Center
}

In Partial Fulfillment

Of the Requirements for the Degree

Master of Dental Science

From The University of Tennessee

By

Bristol Marie Williams

May 2007 


\begin{abstract}
Dental implants are the gold standard of restorative dentistry today. Implants have been used for several years in Orthopedics. Dental implants are an important treatment option for several patients today. When a patient is fully or partially dentate, they are given the opportunity to restore their dentition in ways similar to their natural oral cavity. The past literature states that titanium is one of the first metals used in implant placement. There have been many improvements made on this material. Most modifications have been used in order to promote more bone growth around these implants. Several different coating materials have been used to increase bone formation. Titanium (Ti) surfaces have been coated with hydroxyapatite (HA), plasma spray, and several blasting techniques have been implemented. Tricalcium phosphate (TCP) is a coating that has been used for several years. Due to its porosity and likeness to natural bone, many feel that it is an ideal coating for both dental and orthopedic titanium implants. We believe that TCP coated titanium increases the amount of osteoblastic cell attachment and early bone producing biomarker proteins in osteoblastic cells when compared to HA coated titanium and titanium only materials.
\end{abstract}




\section{Table of Contents}

Chapter 1. Introduction 1

1.1 Bone Composition and Classifications 1

1.2 Osteoinduction and Osteoconduction 2

1.3 Bone Healing and Remodeling 3

1.4 Bone/Implant Interface 3

1.5 Bone/Implant Wound Healing 4

1.6 Implant Surfaces $\quad 4$

$\begin{array}{lr}\text { 1.6.1. Methods and Modifications } & 6\end{array}$

1.6.2. Grit Blasting

$\begin{array}{ll}\text { 1.6.3. Porous Surfaces } & 7\end{array}$

1.7 Hydroxyapatite $\quad 8$

1.7.1. Commercial HA 9

1.7.2. Dissolution HA 9

$\begin{array}{ll}\text { 1.7.3. HA Coatings } & 10\end{array}$

$\begin{array}{ll}\text { 1.7.4. Plasma Sprayed HA Preparation } & 10\end{array}$

1.8 Tricalcium Phosphate Coatings $\quad 12$

1.8.1. Tricalcium Phosphate Resorption 13

$\begin{array}{ll}\text { 1.8.2. Adverse Effects and Indications } & 14\end{array}$

Chapter 2. Research Aims and Objectives 15

2.1 Specific Aims 15

2.2 Hypotheses $\quad 15$

$\begin{array}{ll}2.3 \text { Project Significance } & 15\end{array}$

Chapter 3. Material and Methods 17

3.1 Titanium Substrates $\quad 17$

3.2 Coatings 17

$\begin{array}{ll}3.3 \text { Osteoblast Cell Culture } & 18\end{array}$ 
3.4 Cell Attachment

3.5 Alkaline Phosphatase Activity

3.6 Osteocalcin Assay

Chapter 4. Results

Chapter 5. Discussion

Chapter 6. Conclusion

Chapter 7. Future Studies

List of References

Vita 


\section{Chapter 1. Introduction}

\subsection{Bone Composition and Classifications}

Bone is a connective tissue that consists of a complex composite of cells and extracellular matrix. Bone cells include osteocytes, osteoprogenitor cells, osteoblasts, and osteoclasts. Each of these cells is related to different functions of cell activation, matrix maturation, mineralization, and remodeling. The extracellular matrix contains Type I collagen and ground substance. Ground substance is an aggregate of proteoglycan and noncollagenous glycoproteins that become mineralized. The minerals include calcium and phosphorous and are stored as hydroxyapatite crystals. Through mineralization, bone obtains its rigid structure and function: support and protection of the musculoskeletal system and a physiologic reservoir of calcium and phosphorous. ${ }^{1}$

There are many bone classifications. Dense compact bone forms an outer shell for an inner meshwork of anastomosing spicules of cancellous bone. Woven bone is formed during growth and development and in response to injury. It is a highly cellular osseous tissue with low mineral content, random fiber orientation and minimal strength. It is usually formed at a rate of 3-50um/day or more and is replaced with lamellar bone. Woven bone is essential to initial healing of endosseous implants. Lamellar bone is the mature load bearing bone that comprises most of the adult skeleton. The bone is densely mineralized and highly organized into the following structural units:

- Circumferential lamellae-forms a perimeter of compact bone that encases concentric and interstitial lamellae.

- Concentric lamellae-encases the Haversian canals to form osteons. 
- Interstitial lamellae-irregular shaped remnants of concentric lamellae

All of these units create the Haversian system which encases the neural and vascular tissue of bone. ${ }^{1,2}$

\subsection{Osteoinduction and Osteoconduction}

Bone formation is a complex series of changes that occur leading to newly formed structures. In essence, when an endosseous wound is formed, two stages occur that comprise this wound healing. There is an osteoinduction and osteoconduction portion. These two terms are interrelated but not identical. In the osteoinduction stage, there is a phenotypic conversion of mesenchymal cells into bone-forming cells. ${ }^{3}$ Mesynchymal cells are undifferentiated and they are stimulated to turn into osteoblasts and osteocytes.

Osteoconduction is defined differently than osteoinduction. It is appositional growth permitting bone formation on a surface or down into the pores, channels, or pipes. It relies on the migration of differentiating osteogenic cells to different surfaces (i.e. the implant surface). ${ }^{3}$ Undifferentiated mesenchymal cells migrate to the implant surfaces, attach and proliferate. Different environmental factors determine whether or not mesenchymal cells will differentiate into fibroblasts, chondrocytes, or osteoblasts. Adherence can occur when the cell itself directly binds to the surface or when it binds to arginine-glycine-aspartic acid containing proteins on the surface. At this time, the mesenchymal cells undergo osteoblastic differentiation. Mesenchymal cells are sensitive to surface properties such as surface energy, roughness, and topography. ${ }^{4,5}$ The new osteoblasts produce osteoid which includes matrix vesicles and growth factors. 


\subsection{Bone Healing and Remodeling}

Remodeling is the process of old or damaged bone being replaced by new bone. The rate of bone turnover is approximately $5 \%$ for cortical bone and up to $15 \%$ for trabecular bone. Damaged/old bone is resorbed by osteoclasts which form a cutting cone. Osteoblasts differentiate behind the cutting cone to form a filling cone. Osteoblasts in the filling cone synthesize osteoid which becomes the mineralized osteons. Osteoblasts differentiate into osteocytes during mineralization and maturation of bone. ${ }^{1}$

After implant surgery, bone healing and remodeling is broken into three phases. The first phase is the inflammatory phase. This takes place during the $1^{\text {st }} 10$ days after surgery. At this time, adsorption of plasma proteins, platelet aggregation, clotting cascade activation, cytokine release, nonspecific cellular inflammatory response, and macrophage-mediated inflammation occurs. The second phase is the proliferative phase. This occurs during 3-42 days. At this time, neovascularization, differentiation, proliferation, and activation of cells and production of immature connective tissue matrix is prevalent. The maturation phase is the third phase. This occurs after 28 days. During this time, remodeling of the immature bone matrix with coupled resorption and deposition of bone is prevalent. Remodeling of bone in response to implant loading and physiologic bone recession occurs. ${ }^{1}$

\subsection{Bone/Implant Interface}

The implant/bone interface has been evaluated for several years. The direct bone to implant interface between vital bone and screw-shaped titanium implants was proven to achieve and maintain bone anchorage under loading. This phenomenon is known as 
osseointegration. This term is used to describe the "direct structural and functional connection between ordered, living bone and the surface of a load carrying implant." Branemark and associates coined this term. Branemark also demonstrated that titanium could be predictably implanted and integrated into vital bone. ${ }^{6}$

\subsection{Bone/Implant Wound Healing}

The events of osseous wound healing parallel the healing of bone adjacent to implants. Implant substrate-osteoblast interactions may be characterized as specific, protein-mediated, dynamic, signal-generating events. Implant surface modification may modulate this cell behavior. After implantation, homeostasis, and clot formation, fibrinolysis occurs with the formation of a loose connective tissue stroma that supports angiogenesis. The surface of the implants is conditioned with serum proteins, mineral ions, sugars, lipids, as well as cytokines produced by immune cells. The blood volume around the implant will vary considerably as a function of the implant design and site. The absence of relative paucity of serum proteins such as albumin indicates a selective accumulation or deposition of molecules at the interface. It has been stated that implant surfaces provide anchorage for the fibrin clot to withstand detachment forces during cell migration and thus maintains a pathway for the differentiating osteogenic cells to reach the implant surface. ${ }^{7}$

\subsection{Implant Surfaces}

Implants must be made of a material that is compatible with bone. Accepted materials used in the fabrication of implants are classified into three groups: metals, 
ceramics, and polymers. Each material can be further identified by its biocompatibility as bioinert, biotolerant, and bioactive. Bioinert materials allow close apposition on their surface, leading to contact osteogenesis. Materials considered bioinert include ceramics such as aluminum oxide and zirconium oxide, and metals such as commercially pure titanium and titanium alloys. Biotolerant materials are not rejected when implanted in tissue, but are surrounded by a fibrous layer to form a capsule. Biotolerant metals and polymers encompass gold, cobalt-chromium alloys, stainless steel, zirconium, niobium, tantalum, polyethylene, polyamide, polymethylmethacrylate, and polytetrafluorethylene. Bioactive materials allow for formation of new bone on their surface, exchanging ions to create a chemical bond. Polymers used a bioactive implant materials include hydroxyapatite, tricalcium phosphates, tetracalcium phosphates, calcium phosphates, fluorapatite, brushite, carbon: vitreous and pyrolytic, carbon-silicon and bioglass. Bioactive and bioinert materials are osseoconductive, meaning they form a scaffold for bone ingrowth. ${ }^{8}$

The implant-bone interface is a complex development. There are several factors involved including materials, shape, topography, surface chemistry, mechanical loading, surgical technique, patient bone quality, and quantity. Creating topographic variations from the mean surface plane of the implant can be achieved by additional methods such as titanium plasma spraying, hydroxyapatite (HA) coating, coating with plasma, magnetron sputter coating, calcium phosphate, and other attempts to coat an implant surface with biological molecules. ${ }^{9}$ 


\subsubsection{Methods and Modifications}

Methods of implant surface modifications include abrasion through blasting with titanium oxides or other soluble resorbable biomaterials, grits, or sand-blasting with aluminous oxides, and blasting and acid-attacking or etching with a hydrogen sulfate or hydrogen chloride material. ${ }^{9}$

Some surface treatments include anodizing, cold working (dimpling), sintering, and bead compaction. The surface energy, composition, topography, and roughness of an implant are thought to affect bone formation and apposition.

Some important advantages have been linked to the differences in surface roughness. Implant surface modifications most frequently described in the literature may be divided into: rough surfaces coated with a coating (ex. Titanium plasma-sprayed of hydroxyapatite coated implants). Machine processed (ex. Machined or polished) titanium surfaces without a coating and rough surface implants without a coating (ex. Sand-blasted or acid-etched). These include increases surface area of the implant adjacent to the bone. There has been discussion of improved cell attachment to the implant surfaces, increased bone present at the implant surface, and increased biomechanical interaction of the implant with bone. ${ }^{10}$

After several studies, it has become known that rough-surfaced implants have significantly higher success rates as compared to implants with turned surfaces. The surface roughness of titanium is one factor that helps in determining the balance between bone formation and remodeling at the bone implant interface when compared to TPS and 
machine implants. Bone-implant interface is positively correlated with increasing roughness of the implant surface. ${ }^{10}$

\subsubsection{Grit Blasting}

Titanium oxides and aluminum oxides of various particle diameters are used to abrade the surface of implants. Grit blasting creates a surface of irregular pits and depressions. The particle size, time, pressure and distance create a range of desired surface roughness. ${ }^{8}$ Blast polished titanium implants with 30 and 60 grit aluminum oxide resulted in significantly more bone formation on the implant as well as a higher shear strength at the implant-bone interface as compared to a polished titanium surface alone. ${ }^{8}$

The disadvantage to grit blasting is the potential for particles to remain embedded on the implant surface disrupting osseointegration. ${ }^{11}$ Chemical exposure of a metallic implant to acid etches may enhance osseointegration without adding particulate matter or surface contaminants, eroding pits of specific dimension and shape. ${ }^{8}$ It has been concluded that acid etched implants have a greater resistance to torque removal compared to machined surface titanium. ${ }^{12}$ These results are interpreted as an increase in implant to bone contact.

\subsubsection{Porous Surfaces}

Porous surfaces are differentiated from roughened surfaces by the lack of sharp or jagged topography. Pores are created by sintering spherical powders of metallic or ceramic materials to the metallic core of an implant. The pore size, shape, depth, and volume are affected by the particle size, temperature, and pressure of the sintering 
process. ${ }^{13}$ Maximum bone ingrowth appears to occur at a pour depth of $150-300 \_\mathrm{m} .{ }^{14}$ The pore volume (porosity) directly affects the strength of the sintered coating to contact metal. ${ }^{15}$

\subsection{Hydroxyapatite}

Hydroxyapatite (HA) is composed of calcium, phosphorous oxygen, and hydrogen. The chemical composition is as follows:

$$
\left(\mathrm{Ca}_{10}\left(\mathrm{PO}_{4}\right)_{6}(\mathrm{OH})_{2}\right.
$$

The chemistry of HA lends itself to be similar to the mineral content of hard tissues such as bone, teeth, and vertebrae. ${ }^{1}$ Significant efforts in multidisciplinary research have sought to implicate the use of HA for numerous scientific applications such as separation and purification of proteins, drug delivery systems, and bone implants. ${ }^{25,26,16}$ For each application, the use of the calcium phosphate based material is optimized by specifying its geometry, dimension, density, pore size, mechanical strength, purity, and chemical phase.

HA was developed as coatings for dental and orthopedic implants to combine the bioactive surface HA with the strength of titanium. ${ }^{8}$ The advantages of HA coatings lie within its reported osseoconductive ability to improve the rate and strength of initial implant integration. Enhanced integration could result in earlier stability for prosthetic loading. ${ }^{17}$ 


\subsubsection{Commercial HA}

Commercially available HA grain size is available from $45-160 \mu{ }^{18}{ }^{18}$ However, the large grain size of conventional HA also contributes to its physical weaknesses. Smaller, denser HA feedstocks are the focus of commercial production bioceramics. The size dependent physical properties of materials reduced to the nanoscale can suddenly show very different characteristics compared to what they exhibit on the macroscale, enabling unique applications. ${ }^{19}$

The chemical compositions of HA coating can vary in percent composition of crystalline HA, $\alpha$ or $\beta$-tricalcium phosphates, calcium oxides, calcium pyrophosphates, and amorphous CaP. HA coating crystallinity, phase composition, quantity and type porosity all determine the reactive properties and subsequently the osseointegration potential of the coating. Surface reactions include following a cascade of events including the dissolution of HA, precipitation of the apatite, ion exchange accompanied by absorption and incorporation of biological molecules, cell attachment proliferation and differentiation, and extracellular matrix formation and mineralization. ${ }^{20}$

\subsubsection{Dissolution HA}

The rate of dissolution is associated with the crystallinity of a prepared HA coating. The crystallinity of HA describes the percent of chemical composition in crystalline from within any given HA coating. The rate of dissolution is affected by the ratio of crystalline HA to metastable compounds such as amorphous calcium, calcium oxide, $\alpha$ or $\beta$-tricalcium phosphate, and tetra calcium phosphate within HA that either 
inherent or produced during the application of the coating to an implant substrate. These metastable constituents are more soluble than HA leading to an increase in dissolution. ${ }^{20}$

\subsubsection{HA Coatings}

Plasma-sprayed hydroxyapatite- coated implants are biocompatible and promote early osseointegration by facilitating healing at the cellular level when compared to noncoated titanium implants. This promotion of early osseointegration has been attributed to the increase in strength at the bone-implant surfaces. However, problems with the plasma-sprayed HA coatings have included variation in bond strength at the coatingmetal interface, non-uniformity in coating thickness between vendors, alteration in structural and chemical properties, non-uniformity in coating density. ${ }^{9,20,21}$

One of the greatest concerns about the use of plasma-sprayed HA coated implants is the general lack of long-term documentation on implant survival. Over the years, vast amounts of in vivo research has indicated biocompatibility with HA-coated implants and a better performance when compared to non-coated titanium implants. As a result of these problems, there is a controversy over whether or not HA coatings are beneficial to implant success. However, these problems are not shortcomings inherent in the rationale for HA coating, but rather in the plasma spray technology currently used for depositing HA coatings on implant surfaces. ${ }^{22,23}$

\subsubsection{Plasma Sprayed HA Preparation}

Plasma spraying is the current commercial method for producing HA coatings in implant surfaces. A DC electric arc is struck between two electrodes and an ionized high 
temperature gas (up to $30,000^{\circ} \mathrm{C}$ ) resulted as a stream of gases passing through the electric arc. The increase in gaseous temperature resulted in an expansion of the gas causes the carrier gas stream to pass through the arc approximately the speed of sound. The coating powder, suspended in the carrier gas stream is then fed into the plasma flame. The heat content of the plasma flame and thus the ability to increase the temperature of a particle is strongly dependent on the type of gas used. In addition, the longer the particle remains in the plasma flame, the higher will be the plasma temperature. Ideally, only a thin outer layer of each powder particle gets into the molten plastic state which undergoes some phase transitions. This molten plastic state is critical to ensure an adhesive and dense coating. As a result, properties of the plasma sprayed coatings is highly dependent on particle size, type of gas used, speed of the plasma, the distance between the implants and plasma nozzle, and the cooling rate for the desired structural and chemical properties..$^{22}$

Apatite is known to be thermodynamically unstable during plasma spraying at high temperatures. When HA is plasma sprayed in air, calcium oxide is usually formed and its concentration will affect the integrity of the plasma-sprayed HA coatings. A poor coating integrity is resulted from high concentration of calcium oxide reaction with water. In addition, an increase in calcium oxide can also be resulted from the presence of a gas carrier resulted in a lower flame velocity and a more flame enthalpy as compared to the use of nitrogen gas, thereby resulting in more melting and decomposition of HA particles. Plasma-sprayed HA coatings usually have a thickness of 50-100_m. It is desirable to have a plasma-sprayed coating that is completely dense and consists of pure 
HA. As such, the $\mathrm{Ca} / \mathrm{P}$ ration in the plasma-sprayed coating should be as similar to natural HA. ${ }^{23}$

Aside from the weak coating-metal interface, vast amounts of investigations have indicated that there are no accepted standards for producing HA coatings on implant surfaces and that all HA coatings are not identical. Variability in coating quality has resulted in many conflicting animal and clinical observations. Although plasma-sprayed HA coatings on implant surfaces have been indicated to enhance implant stability, such findings have not been universal. Typical plasma-sprayed HA coatings have been reported to consist primarily of partially dehydrated HA, with amorphous calcium phosphate and other more soluble phases such as TCP produced during the hightemperature deposition process. Although plasma spraying has the ability to produce coating crystallinity in the range of $30-70 \%$ the crystallinity for plasma-sprayed HA coatings produced under normal deposition process are approximately $65 \%$. In addition, the use of plasma spraying technology often results in coating properties that differs from the starting HA powders. ${ }^{23,24}$

\subsection{Tricalcium Phosphate Coatings}

TCP is a scaffold which was approved by the FDA in the late 1900's for use in traumatic or surgical-created defects in the extremities, pelvis, and spine. Small crystals TCP (70-100nm) are formed into a 90\% scaffold. In the fully-interconnected polyphorous structure, $75 \%$ of the pores range from $100-1000 \mu \mathrm{m}$. The remaining $24 \%$ range from $1-100 \mu \mathrm{m}$. The macroporosity provides space in which bone in growth can occur by osteoconduction. The smaller pores may be less available for tissue ingrowth, 
but significantly enhance fluid flow and diffusion through the matrix, which may improve the metabolic environment for cells within the matrix's. The microporosity also provides a vehicle for promoting uptake of fluid and cells into the matrix capillary action when the matrix is contacted with blood or marrow. The porosity and the nanocrystalline composition enhance dissolution and cell-mediated resorption after implantation and during the bone remodeling. Similar to interporous hydroxyapatite, the architecture of the $\beta$-TCP scaffold resembles native trabecular bone. ${ }^{25}$

\subsubsection{Tricalcium Phosphate Resorption}

The resorption characteristics of TCP are consistent with data collected for this material after in vivo implantation more than 25 years ago. The resorption rate of scaffold TCP is intended to match the course of natural bone healing after implantation. ${ }^{25}$

Pre-clinical evaluations have characterized the biologic performance of this porous scaffold. After implantation, the high porosity of the TCP allows rapid infiltration by local cells and fluids. In large metaphyseal defects of the canine humerus, quantitative histomorphometric analysis showed the formation of bone through the porous scaffold in conjunction with tricalcium resorption. Significant bone formation was reported throughout the implant as early as 3 weeks. At 12, 24, and 52 weeks, the new bone in the defect remodeled into a highly interconnected bone tissue indistinguishable from normal adjacent bone. TCP resorption paralleled with bone in growth, with 76\% resorbed at 6 weeks and $86 \%$ resorbed at 12 weeks. At 52 weeks, less than $2 \%$ remained. $^{25}$ 


\subsubsection{Adverse Effects and Indications}

To date, no adverse events of failures have been observed. However, no peerreviewed and published reports describing clinical efficacy are available. The surface area and porosity provided by TCP may make it an effective carrier for cells derived from bone marrow and other sources. Tricalcium phosphate maybe a useful carrier or delivery system for some biologic and pharmaceutical agents, such as bone morphogenic proteins and antibiotics. ${ }^{25}$ 


\section{Chapter 2. Research Aims and Objectives}

\subsection{Specific Aims}

- Determine the amount of cell attachment to the TCP-coated and HA-coated surfaces in comparison to Ti (control).

- Determine the osteocalcin levels on the TCP-coated and HA-coated surfaces in comparison to $\mathrm{Ti}$ (control).

- Determine the alkaline phosphatase specific activity on the TCP-coated and HA coated surfaces in comparison to Ti (control).

\subsection{Hypotheses}

- TCP-coated titanium will cause an increase in cell attachment when compared to HA-coated titanium and Ti (control).

- TCP-coated titanium will cause an increase in alkaline phosphatase activity when compared to HA-coated titanium and Ti (control).

- $\quad$ TCP-coated titanium will cause an increase in osteocalcin production when compared to HA-coated titanium and Ti (control).

\subsection{Project Significance}

HA and TCP coatings were produced using a modified sol-gel process and spincoated on Ti surfaces. The chemical composition and crystallinity of the applied coating was precisely controlled over a wide range of values, permitting optimization of the coating for the promotion of osseointegration. The coatings were deposited as thin films 
from $1-5 \mu \mathrm{m}$ thick using this innovative process, much thinner than coatings were applied using plasma spraying technology and thus having a higher adhesive strength. The solgel coating process is not a line of sight process and thus allows a uniform coating to be applied to roughened or beaded commercial dental implant surfaces. 


\section{Chapter 3. Material and Methods}

\subsection{Titanium Substrates}

Commercially pure grade 2 titanium (Ti) from Metal Samples, Munford, AL (14 $\mathrm{mm}$ diameter and $2 \mathrm{~mm}$ thick) were used in this study. The Ti surfaces were wet ground with 240, 400 and 600 grit silicon carbide paper. Surface roughness measurement using Surtornic 3 profilometer (Taylor-Hobson, UK) indicated a $R_{a}$ value of $0.37 \pm 0.01 \mu \mathrm{m}$. After grinding, the Ti surfaces were degreased and cleaned by ultrasonically immersing them in acetone and ethanol for 10 minutes each. This was followed by deionized water rinsing between applications of each solvent. Following degreasing and cleaning, the Ti surfaces were passivated by exposing the surfaces to a $40 \%$ volume nitric acid solution at room temperature for 30 minutes (ASTM F86-76). All surfaces were rinsed with deionized water after passivation and air-dried. The non-coated Ti surfaces were then used as controls or be subject to hydroxyapatite (HA) or tricalcium phosphate (TCP) coatings.

\subsection{Coatings}

In this study, HA and TCP sol were used as coatings on Ti implant surfaces. The HA or TCP sol was prepared by reacting calcium nitrate tetrahydrate $\left[\mathrm{Ca}\left(\mathrm{NO}_{3}\right)_{2} \cdot 4 \mathrm{H}_{2} \mathrm{O}\right]$ (Aldrich) with the triethyl phosphite $\left[\left(\mathrm{OC}_{2} \mathrm{H}_{5}\right)_{3} \mathrm{P}\right]$ (Fluka) in methyl alcohol using correct amounts to obtain the stoichiometric $\mathrm{Ca} / \mathrm{P}$ ratio. This was followed by the addition of $0.03 \mathrm{~mol}$ of $\mathrm{HCl}$ and $0.1 \mathrm{~mol}$ of DCCA (Drying Control Chemical Additive). All work was performed in argon atmosphere. The mixed solution was then aged at $80^{\circ} \mathrm{C}$ for 12 
hours. The aged solution was filtrated through a $0.2 \mathrm{M}$ syringe filter, and then be aged again at $50^{\circ} \mathrm{C}$ for 7 days. After aging, $\mathrm{HA}$ or TCP coatings was then spin-coated on $\mathrm{Ti}$ surfaces at $3500 \mathrm{rpm}$ for 30 seconds. The coated-Ti surfaces were immediately dried at $70^{\circ} \mathrm{C}$ for 12 hours and then heat-treated at $650^{\circ} \mathrm{C}$ for 3 hours. All samples were autoclaved prior to culture experiments.

\subsection{Osteoblast Cell Culture}

The osteoblast cell culture study was performed with the ATCC CRL 1486 human embryonic palatal mesenchyme cell line (HEPM), a precursor of osteoblast cells. The cells were suspended in Dulbecco's modified Eagle's medium (DMEM) containing 10\%

FBS, L-ascorbic acid, $\alpha$-glycerophosphate, $2.5 \times 10^{-9} \mathrm{M} 1,25(\mathrm{OH})_{2}$ vitamin $\mathrm{D}_{3}$ and a $1 \%$ antibiotic/antimycotic mixture of penicillin, streptomycin and fungizone. Experiments were conducted in a $5 \% \mathrm{CO}_{2}$ humidified incubator $37^{\circ} \mathrm{C}$, and the medium was changed twice a week. Sterilized HA (controls), Ti (controls), and Tri-calcium phosphate coated surfaces were placed at the bottom of a 24-well plate. Samples were subsequently assayed for cell attachment, proliferation activity, and alkaline phosphatase activity. Human embryonic palatal mesenchyme cells were used for determining the total protein production, alkaline phosphatase specific activity, and 1,25(OH)2 vitamin D3 stimulated osteocalcin production on different Ti surfaces. Sterilized surfaces were placed at the bottom of a well of 24 -well plate. One $100 \mathrm{ml}$ of each osteoblast cell suspension was seeded into each well. Four replicates were conduced for each experimental point. The cultures were assayed at $0,4,8$, and 12 days. 


\subsection{Cell Attachment}

At 30, 60, 120, and 180 minutes after seeding, cells attached on each surface were evaluated. A $200 \mu \mathrm{l}$ cell suspension (44,000 cells) were seeded on each surface. At each time point, triplicate samples from each group were assayed by removing the media and washing the samples twice with PBS. The total volume of PBS used for each was $200 \mu$ l. The removed media and washed solution was saved and recorded as total volume. The number of unattached cells in the removed media and wash solution were subsequently determined using Beckman Z2 Coulter Counter. Cells attached on each surface were measured by subtracting the unattached cell concentration from the initial cell concentration seeded. Mean attachment cell concentrations between the different surfaces were statistically analyzed using the ANOVA, with the differences compared using Tukey's procedure. The alpha level for data analysis was set at 0.05 , and differences was considered significant if $\mathrm{p}<0.05$.

\subsection{Alkaline Phosphatase Activity}

Alkaline phosphatase (ALP) activity was quantified by rinsing (twice) the cells with PBS, followed by exposing the cells to $0.2 \%$ Triton-X-100 solution in order to permeabolize the cell membranes. The cells were then subjected to three freeze/thaw cycles, ultrasonically homogenizing the samples for 15 minutes, and centrifuging to remove large cellular debris. The resulting supernatant was assayed for the release of $\mathrm{p}$ nitrophenol from $\mathrm{p}$-nitrophenylphosphate $(\mathrm{pH}=10.2)$ and its specific activity calculated $50 \mu 1$ of p-mitrophenyl phosphate substrate was added. Followed by incubation in the dark at room temperature for 1 hour. After stopping the reaction using $50 \mu \mathrm{l}$ of $1 \mathrm{M} \mathrm{NaOH}$, 
and the absorbance was read at $405 \mathrm{~nm}$ using microplate readers. ALP activity of cells from each surface were then calculated from prepared standards (Sigma, St. Louis, MO). Results were calculated as nmol p-nitrophenol per hour and normalized to DNA as measured by PicoGreen assay kit (Molecular probes Eugene, OR). Data was plotted as the normalized ALP activity at 3, 6, 9, and 12 days. Significant differences in protein levels were statistically evaluated using ANOVA with Student Newman Kuels procedure as the post hoc test for evaluation of significant differences at $\mathrm{p}<0.05$.

Alkaline phosphatase specific activity was assayed as follows: a $50 \mu$ l aliquot of the same cell lysate used for protein determination was added to $50 \mu$ l of working reagents in a 96 well culture plate and incubated at $37 \% \mathrm{C}$ for $1 \mathrm{hr}$. The working reagent consists of 1.5 M 2-amino-2 methyl-propanol (Sigma), $20 \mathrm{mM}$ p nitrophenol phosphate (Sigma) and $1 \mathrm{mM}$ magnesium chloride (1:1:1). The reaction was stopped using $100 \mu \mathrm{l}$ of $1 \mathrm{~N}$ sodium hydroxide and the absorbance read at 4-5nm using a microplate reader. Alkaline phosphatase specific activity was calculated using the previously determined protein values. Significant differences in protein levels was statistically evaluated using ANOVA with Student Newman0Kuels procedure as the post hoc test for evaluation of significant differences at $\mathrm{p}<0.05$.

\subsection{Osteocalcin Assay}

Osteocalcin production was assayed as follows: At the experimental time point, media was removed and replaced with media containing $2.5 \times 10^{-9} \mathrm{M} 1,25(\mathrm{OH}) 2$ vitamin D3. After 24 hours, the media was replaced again with media containing the $1,25(\mathrm{OH}) 2$ vitamin D3. After 48 hours, the media was collected and frozen at $-20^{\circ} \mathrm{C}$. The samples 
were then lyophilized using the speed-vac (Savant). The 1,25 (OH)2 vitamin D3 osteocalcin production was measured using commercially available rat osteocalcin radioimmunoassay reagents (Biomedical Technologies, MA). The samples $(25 \mu 1)$ was added to the $100 \mu l$ osteocalcin antiserum. They were covered tightly and incubated at $37^{\circ} \mathrm{C}$ for $2.5 \mathrm{hrs}$. The samples were then be aspirated completely and washed 3 times with $.3 \mathrm{~mL}$ phosphate-saline was buffer. Then $100 \mu 1$ Streptavidin-Horseradish Peroxidase reagent was added to each well. The wells were incubated at room temperature for 30 minutes. One volume of TMB solution (peroxidase substrate) with one volume of hydrogen peroxide solution (BT-468) was mixed and set aside. The plates were washed as before and $100 \mu l$ of substrate mix was added to each well, incubated at room temperature in the dark, for 15 minutes. $100 \mu 1$ of stop solution was added and absorbance was measured immediately at $450 \mathrm{~nm}$. The radioactive count of the samples was correlated to a standard known osteocalcin concentration curve and differences in 1,25 $(\mathrm{OH}) 2$ vitamin D3 stimulated osteocalcin production was statistically compared using the ANOVA test, with the Student Newman-Kuels procedure as the post-hoc test for the evaluation of significant differences at $\mathrm{p}<0.05$. 


\section{Chapter 4. Results}

Initial osteoblast attachment study was performed on titanium (control, HA coated, and TCP coated surfaces. As shown in Figure 4.1 at 30 minutes, HA surfaces exhibited the lowest amount of osteoblast attachment, whereas Ti surfaces exhibited highest amount of osteoblast attachment and TCP coated had the second highest attachment percentages. While the amount of cell attachment levels rose exponentially, by 180 minutes.

After evaluating cell attachment at different times, it was discovered that there is a significant difference between the cell attachment percentages at 30 minutes. During this time, the significant difference is largest between HA and titanium (control) surfaces. There is a larger amount of cell attachment to titanium at this time as opposed to HA coated titanium. There is also a significant difference between HA coated and TCP coated titanium at this time. There is more cell attachment to TCP coatings than HA coated titanium surfaces.

At 60 minutes, there is a significant difference in cell attachment on the titanium surfaces. The difference in coatings is evident when comparing Ti (control) and HA coated titanium. There is a higher amount of cell attachment to Ti (control) than HA coated titanium.

At 120 minutes, there is no significant difference between the control or the coated surface titanium. There is, however, a significant difference between the HA coatings when compared to the TCP coated surfaces. There is more cell attachment to the TCP coatings than the HA coatings. When comparing the HA and Ti (control), there 


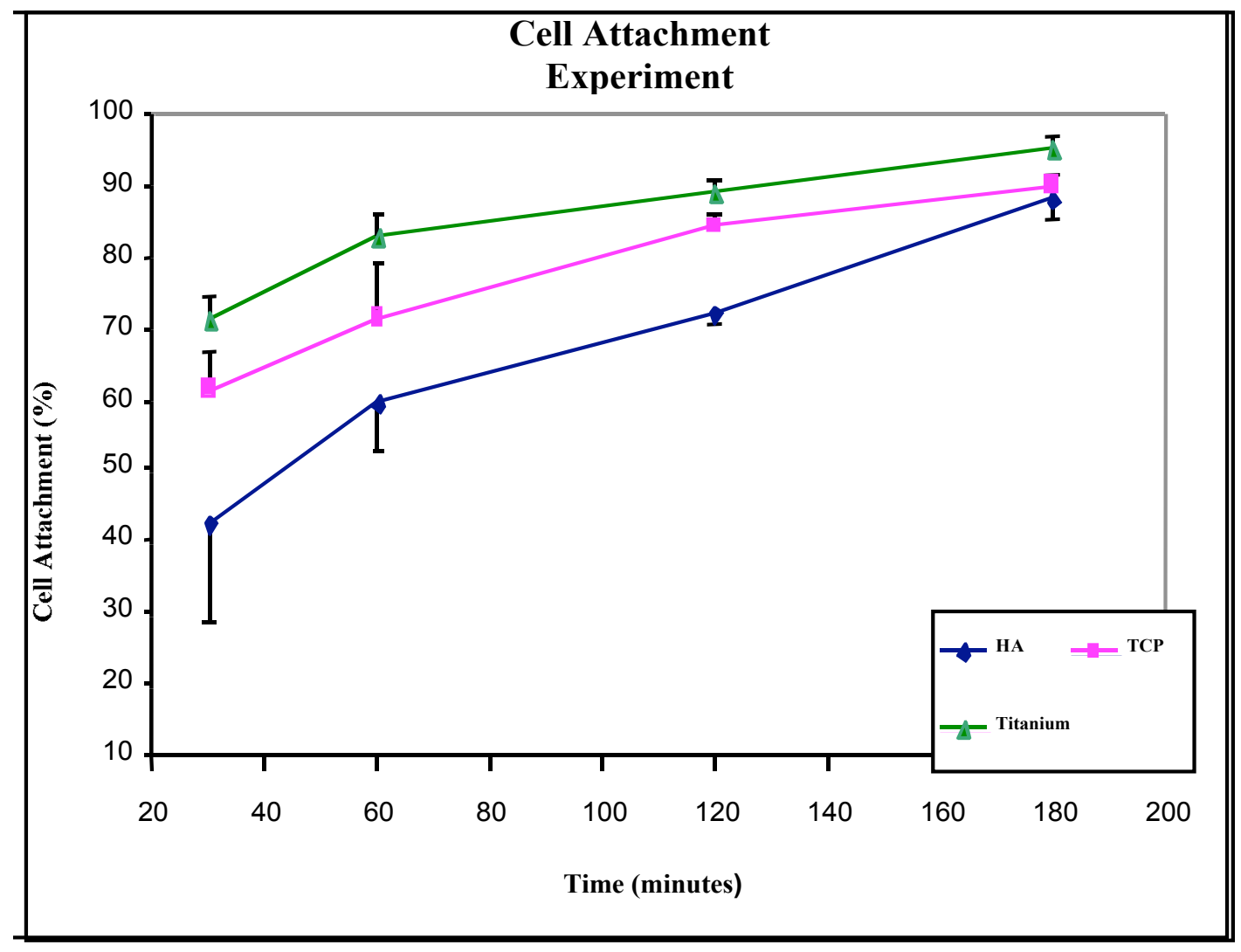

Figure 4.1: Initial Cell Attachment Study on Hydroxyapatite and Tricalcium Phosphate Surfaces over 180 Minutes. 
is a significant difference between the two. There is more attachment on the Ti (control) surface than the HA coated surface. When comparing the Ti (control) and TCP coated surfaces, there is a significant difference between the two. There is more cell attachment on the Ti (control) surface than the TCP coated surface.

At 180 minutes, there is a significant difference between the Ti (control), TCP coated, and HA coated titanium. When comparing the Ti (control) and HA coated surface, there is a significant difference between the two. There is a higher amount of cell attachment on the Ti (control) surface than the HA coated surface. When comparing the Ti (control) and TCP coated surface, there is a significant difference between the two groups. There is a higher amount of cell attachment on the Ti (control) surface than the TCP coated surface.

Early alkaline phosphatase production is evaluated in Figure 4.2. On day 3, there is a significant difference in ALP activity the Ti (control), HA coated, and TCP coated surfaces. When comparing HA and TCP coated surfaces, there is a significant difference between both groups. There is a larger amount of ALP production in HA coated surfaces as opposed to TCP coated surfaces. When comparing Ti (control) and HA coated surfaces, there is a significant difference between the two groups. There is larger amount of ALP production in HA as opposed to Ti (control) surfaces.

On day 6 , there is no significant difference between al three groups. There is no significant difference when the groups are compared to each in pairs (i.e. HA vs TCP, HA vs Ti (control), etc). 


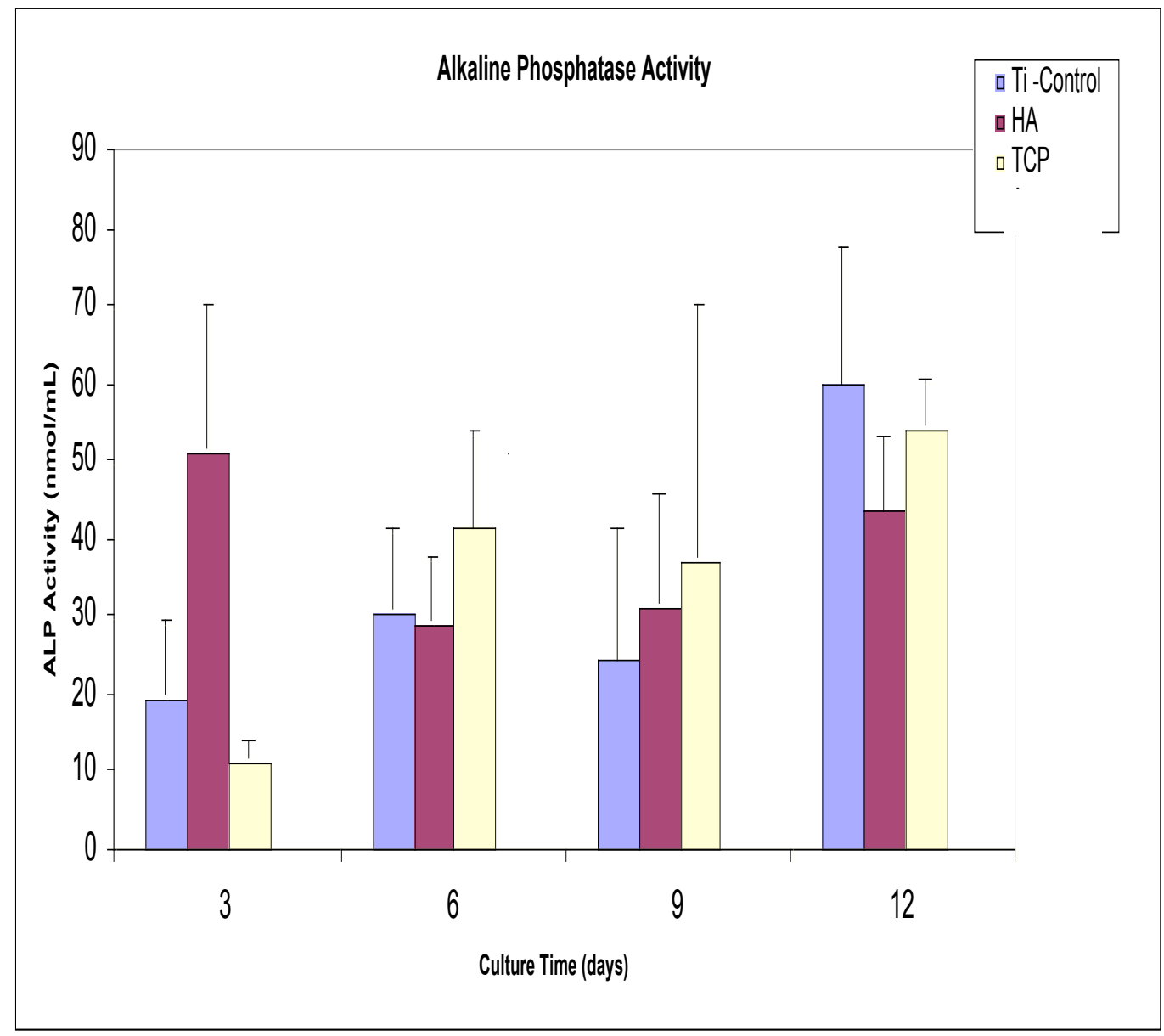

Figure 4.2: Alkaline Phosphatase Activity. 
On day 9, there is no significant difference between al three groups. There is no significant difference when the groups are compared to each in pairs (i.e. HA vs TCP. HA vs Ti (control), etc).

On day 12 , there is no significant difference between al three groups. There is no significant difference when the groups are compared to each in pairs (i.e. HA vs TCP. HA vs Ti (control), etc).

Osteocalcin production on all three surfaces is evaluated in Figure 4.3. On day 3, there is no significant difference between all three groups when observing osteocalcin levels. There is no significant difference when the groups are compared to each in pairs (i.e. HA vs TCP. HA vs Ti (control), etc).

On day 6 , there is no significant difference between all three groups. There is no significant difference when the groups are compared to each in pairs (i.e. HA vs TCP. HA vs Ti (control), etc).

On day 9 , there is no significant difference between all three groups. There is a significant difference between Ti (control) and TCP coated surfaces. There is a larger amount of osteocalcin production in Ti (control) when compared to TCP coated surfaces.

On day 12 , there is no significant difference between all three groups. There is no significant difference when the groups are compared to each in pairs (i.e. HA vs TCP. HA vs Ti (control), etc). 


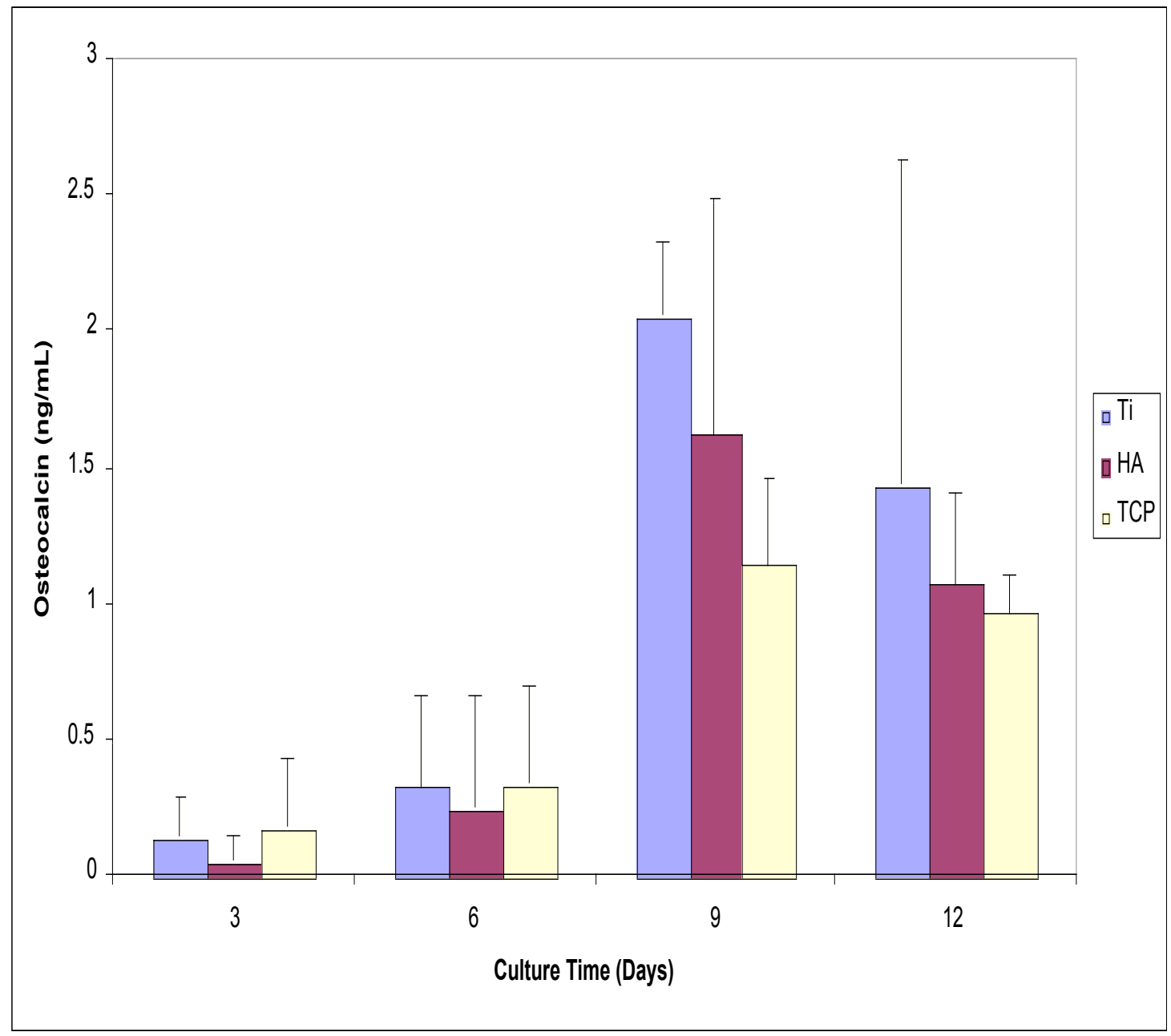

Figure 4.3: Osteocalcin Activity. 


\section{Chapter 5. Discussion}

After evaluating the HA, TCP, and Ti (Control) materials, it is evident that the TCP coated titanium does not induce larger amounts of osteoblastic proteins production in the early stages of osseointegration. During the cell attachment study, it became evident that during the first 20-40 minutes cell attachment percentages tend to be highest in the titanium with no coating and lowest in HA coated surfaces. As the minutes progress, both test groups and the controls tend to have the same percentage of cell attachment.

The coatings and preparations on the titanium disks may play a role in the cell attachment percentages. Cell attachment of osteoblasts to titanium depends on the surface roughness of the material. It has been proven that osteoblasts attach more readily to roughed surfaces (sandblasted and sandblasted/acid-etched) compared to polished or acid-etched surfaces alone. Roughened surfaces alone can support cell attachment of osteoblasts proving that the surface alone is capable of controlled cell attachment. ${ }^{28}$ In this study, Titanium was wet ground and left without a coating as opposed to the HA and TCP coated titanium. The roughened surface of the uncoated titanium allows for the earlier initial cell attachment. As the minutes progress, HA and TCP increase in cell attachment percentages, but they never equal to uncoated titanium attachment levels.

Osteoblasts are rich in alkaline phosphatase. While the function of alkaline phosphatase (ALP) is unknown, it has been suggested that is plays a major role in bone mineralization of newly formed bone. Measurements of total serum ALP is useful when 
the amount from bone is exceptionally high and concentrations from other sources are not increased and are stable. $^{29}$

ALP activity was measured and compared between HA, TCP , and uncoated titanium controls. When (ALP) levels were evaluated, at day 3 HA has the highest amount of ALP activity. By day 12, uncoated titanium has the highest level of (ALP) levels. The increase in the uncoated titanium may again be due to the roughed surface and increase osteoblast attachment to this material. At 12 days, ALP on $\beta$-TCP coatings have increased. This may be attributed to the fact that in several other studies it has been proven that there is minimal bone ingrowth on calcium phosphate treated material. It has been shown that at 2 weeks, the strength of fixation to this material begins to increase. ${ }^{30}$ This may explain why there is such an increase in ALP attachment in TCP as we get closer to 2 weeks.

Osteocalcin activity is a key biomarker in osteoblast production. Osteocalcin is the major noncollagen protein of the bone matrix. The function of osteocalcin is not clear, but it is thought to serve as a site for hydroxyapatite crystal. In the process of the matrix synthesis, some osteocalcin is released and circulates in blood with a short halflife determined mainly by renal clearance. Although no intact osteocalcin is released during bone resorption, fragments are released in vitro and also during resorption and formation. $^{29}$

During the osteocalcin study, at 3 days, TCP had the largest amount of osteocalcin production. By day 12, uncoated titanium has the highest amount of osteocalcin production but all three materials have had a decrease in osteocalcin production overall. This may be due to the fact that more osteoclasts are beginning to 
form around day 12. With this being the case, there has been a decrease in osteocalcin formation. Another factor may be attributed to the dissolution rates of TCP and HA. Over time, there tends to be a continual increase in the calcium and inorganic phosphorous in HA and TCP. The additional increase of inorganic phosphorous has been suggested to slow down osteoblast differentiation and mineralization. ${ }^{31}$ 


\section{Chapter 6. Conclusion}

In summary, during early cell attachment, there is a higher amount of cell attachment in Ti (control) surfaces as opposed to the other tested surfaces. As the test progressed, all surfaces showed an increase in cell attachment, but Ti (control) had the highest amount of cell attachment.

When evaluating ALP production, at day 3, HA coated surfaces had the highest amount of ALP production. As days continued, there was no significant difference between any of the tested groups.

When evaluating osteocalcin production, there was no significant increase in the amount of production between any of the groups until day 9. At day 9. Ti had a much larger increase in the amount of osteocalcin production than TCP. At the end of the study, there was no major difference between any of the groups.

It was thus concluded from this study that TCP coatings did not increase cell attachment, alkaline phosphatase production, or osteocalicn production in early adjcaent bone formation when evaluating osteoblast cells. 


\section{Chapter 7. Future Studies}

In the future, more studies should be conducted using uncoated titanium (control) comparing it to HA and TCP. Suggested modifications for future studies are as follows :

- TCP concentrations should be evaluated at different concentration levels. While the same mediating factors are evaluated, it may be discovered that higher levels of TCP amount to higher levels of cell attachment, ALP production, and osteocalcin production. .

- The time period of the experiments need to be extended. The cell attachment needs to be evaluated at periods longer than 180 minutes. ALP may need to be evaluated for 24 days. Osteocalcin production needs to be evaluated for 24 days..

- Ti (control) surfaces need to be sand blasted and evaluated as opposed to wet grinding the surfaces.

- More cell markers need to be evaluated. Instead of looking at osteocalcin, osteopontin production should be evaluated.

- Osteoclast cells need to be evaluated due to the fact that bone formation is also dependent on bone resorption.

- All three of these surfaces should be evaluated in animal studies. 


\section{List of References}

1. Whitson SW. Oral Histology: Development, Structure, and Function. St. Louis: Mosby Year Book, 1987. 104-127.

2. Ross MH, Romrell LJ, Kaye GI. Histology: A Text and Atlas. Baltimore: Lippincott, 1995. 150-187.

3. Davies J.E. et al. Mechanisms of endosseous integration. Int J Prosthodont 1998; 11(5): 391-401.

4. Roberts W.E., Turley P.K., Brezniak N., Fielder P.J. et al. Bone physiology and metabolism. CDA J 1987; 15(10): 54-61.

5. Raghavendra S., Wood M., Taylor T. et al. Early wound healing around endosseous implants: A review of the literature. Int J Oral Maxillofac Implants 2005; 20(4): 425431.

6. Albrektsson T, Branemark PI, Hansson HA, Linderstrom J. Osseointegrated titanium implants. Requirements for ensuring a long-lasting, direct bone-to-implant anchorage in man. Acta Orthop Scand 1981; 52(2): 155-70

7. Davies J.E. et al. Understanding peri-implant endosseous healing. J Dent Educ 2003; 67(8): 932-949.

8. Sykaras N, Iacopino AM, Marker VA, Triplett RG, Woody RD. Implant materials, designs, and surface topographies: Their effect on osseointegration. A literature review. Int J Oral Maxillofac Implants 2000; 15(6): 675-690.

9. Ong J.L. and Chan D.C. Hydroxyapatite and their use as coatings in dental implants: A review. Crit Rev Biomed Eng1999; 28(5\&6): 1-41.

10. Feighan J.E., Goldberg V.M., Davy D., Parr J.A. et al. The influence of surfaceblasting on the incorporation of titanium-alloy implants in a rabbit intramedullary model. J Bone Joint Surg Am 1995; 77(9): 1380-1395.

11. Orsinis G, Assenza B, Scarano A, Piatelli M, Piatelli A. Surface analysis of machined versus sandblasted and acid-etched titanium implants. Int J Oral Maxillofac Implants 2000; 15(6): 779-84.

12. Chen CC, Huang TH, Kao CT, Ding SJ. Characterization of functionally graded hydroxyapatite/titanium composite coatings plasma-sprayed on ti alloys. J Biomed Mater Res B Appl Biomater 2006; 78(1): 146-52. 
13. Pilliar RM. Overview of surface variability of metallic endosseous dental implants: textured and porous surface-structured designs. Implant Dent 1998; 7(4): 305-14.

14. Bobyn JD, Pilliar RM, Cameron HU, Weatherly GC. The optimum pore size for the fixation of porous-surfaced metal implants by the ingrowth of bone. Clin Orthop Relat Res 1980; 150: 263-70.

15. Hoffmann AA, Bloebaum RD, Bachus KN. Progression of human bone ingrowth into porous-coated implants. Rate of bone ingrowth in humans. Acta Orthop Scand 1997; 68(2): 161-6.

16. Koutsopoulos S. Synthesis and characterization of hydroxyapatite crystals: A review study on the analytical methods. J Biomed Mater Res 2002; 62(4): 600-12.

17. Jarcho M. Retrospective analysis of hydroxyapatite development for oral implant applications. Dent Clin North Am 1992; 36(1): 19-26.

18. Zheng X, Huang M, Ding C. Bond strength of plasma-sprayed hydroxyapatite/ti composite coatings. J Biomed Mater Res 2000; 21(6): 841-849.

19. Salata O. Applications of nanoparticles in biology and medicine. J Nanobiotechnology 2004; 2(1): 3.

20. Xue W, Liu X, Zheng X, Ding C. Effect of hydroxyapatite coating crystallinity on dissolution and osseointegration in vivo. J Biomed Mater Res 2005; 74(4): 553-61.

21. Hacking S.A. et al. Relative contributions of chemistry and topography to the osseointegration of hydroxyapatite coatings. Clin Orthop Relat Res 2002; 405: 24-38.

22. Meffert R.M., Langer B., and Fritz M. Dental implants: A review. J Periodontol 1992; 63: 859-870.

23. Yang Y., Ong JL. et al. Plasma-sprayed hydroxyapatite-coated and plasma-sprayed titanium-coated implants. Biomater Orthop 2004; 26: 401-423.

24. Kim Y., Koak J., et al. A histomorphometric analysis of the effects of various surface treatment methods on osseointegration. Int J Oral Maxillofac Implants 2003; 18(3): 349-356.

25. Wahl DA, Czernuszka JT. Collagen-hydroxyapatite composition for hard tissue repair. Eur Cell Mater 2006; 11: 43-56.

26. Suen RB, Lin SC, Hsu WH. Hydroxyapatite-based immobilized metal affinity adsorbents for protein purification. J Chromatogr A 2004; 1048(1): 31-9. 
27. Bucholz RR et al. Nonallograft osteoconductive bone graft substitutes. Clin Orthop Relat Res 2002; 395: 44-52

28. Keller J.C., Schneider G.B., Galen B. Effects of implant microtopography on osteoblast cell attachment. Implant Dent 2003; 12(2): 175-181

29. Watts N B. Clinical utility of biochemical markers for bone remodeling. Clin Chem 1999; 45: 1359-1368.

30. Rivero D.P., Fox A.K, Skipor R.m. Calcium phosphate-coated porous titanium implants for enhanced skeletal fixation. J Biomed Mater Res 1998; 22(2): 191-201.

31. Ma S., Yang Y., Carnes D.L., et al. Effects of dissolved calcium and phosphorous on osteoblast responses. J Oral Implant 2005; 31(2): 61-67. 


\section{Vita}

I was born in Kansas City, Missouri on February 5, 1979. I earned my B.A. in English from Xavier University in New Orleans, LA. I received my Doctorate in Dental Science from Meharry Medical College School of Dentistry in Nashville, TN. I am currently attending The University of Tennessee Health Science Center receiving my certificate in Periodontology and my Masters in Dental Science. 